Claudia Hillebrandt, Sonja Klimek, Ralph Müller, Rüdiger Zymner

\title{
Einleitung: Wer spricht das Gedicht?
}

\section{Adressantenmarkierung in Lyrik}

Das noch junge Forschungsfeld der Lyrikologie lässt sich durch die Zielsetzung charakterisieren, die Lyrik als eigenständig bestimmbare und nicht an die Grenzen von einzelnen Nationalliteraturen gebundene Gattung theoretisch reflektiert, methodisch geklärt, historisch informiert und in diesem Sinne systematisch zu erforschen (vgl. hierzu vertiefend Zymner 2009, 7-9; von Ammon 2015). Der vorliegende wie auch der zweite Band Grundfragen der Lyrikologie $e^{1}$ verstehen sich als Beiträge in Richtung einer solchen Lyrikologie.

Im vorliegenden Band steht ein in lyrikologischen Beiträgen schon länger diskutiertes Problem der Lyriktheorie und -analyse im Zentrum, das wir hier vorläufig das >Problem der Äußerungsinstanz` nennen möchten. Es wird unter anderem noch einmal zu prüfen sein, ob und inwiefern aus schon häufiger angeführten fiktionstheoretischen wie auch aus in diesem Zusammenhang bisher wenig beachteten modalitätsspezifischen Gründen geeignetere Konzepte und Begriffe zu Erfassung und Benennung von `Äußerungsinstanzen` der Lyrik zu entwickeln sind. Terminologische Vorschläge, um das Problem der `Äußerungsinstanzen anhand des Begriffspaars `Adressant ২ - >Adressat $\mathrm{zu}$ rekonstruieren, legen wir am Ende dieser Einleitung vor. Wie diese sich in eine mögliche lyrikologische Begriffssprache einfügen lassen, verdeutlicht das Glossar von Rüdiger Zymner, das auf diese Einleitung folgt. Die Beiträgerinnen und Beiträger sind von uns eingeladen worden, sich auf diese terminologischen Vorschläge zu beziehen, sei es zur kritischen Übernahme, sei es zur Abgrenzung und Profilierung der jeweils eigenen Begrifflichkeit.

Über terminologische Überlegungen hinaus sollen die Beiträge in diesem Band deutlich machen, dass die Frage nach der Personalität von >Äußerungsinstanzen` (und damit auch ihrer Analyse), die bisher zugunsten begrifflicher

1 Eine weitere, vertiefte Auseinandersetzung mit zentralen Fragen der Lyrikologie wird der Fortsetzungsband bieten, der momentan vorbereitet wird. Im Rahmen eines DFG-finanzierten Netzwerks werden dabei die Themen (1) typische Merkmale der Lyrik, (2) Funktionen der Lyrik, (3) lyrische Zeitkonzepte, (4) mündliche bzw. schriftliche Formatierung von Lyrik, (5) Methoden der Lyrikanalyse sowie (6) Lyrik und Raum behandelt. 
Diskussionen in den Hintergrund getreten ist, breitere lyrikologische Aufmerksamkeit verdient. Um das Problem der `Äußerungsinstanz in seinen verschiedenen Facetten deutlich werden zu lassen, gehen wir von einer nur scheinbar harmlosen Frage aus: Wer spricht im Gedicht? Ist es der Dichter? Das >lyrische Ich «? Das `Textsubjekt`? Oder ist die `Äußerungsinstanz` im Gedicht gar eine Unbestimmtheitsstelle des Textes, die Rezipienten beliebig ausfüllen können, indem sie sich entweder den Dichter, sich selbst oder eine fiktive Figur vorstellen? Vielleicht spricht - so Robert Gernhardt (2005, 134, V. 20) - gar »ein Wesen mui [!] generis«? Diese Frage, von der die in diesem Band versammelten Beiträge ausgehen, ${ }^{2}$ führt mitten in grundlegende Probleme der Lyrikologie. Denn abgesehen vom Fragepronomen swer, das auf eine »irgendwie« geartete personale Instanz verweist, werden bei näherem Hinsehen auch die übrigen Wörter dieses Satzes fragwürdig:

'Sprechen`? Die Festlegung auf gesprochene Sprache erscheint angesichts der Tatsache, dass uns viele Gedichte in gedruckter Form begegnen und zum Beispiel Figurengedichte überhaupt nur im Medium der Schrift funktionieren, durchaus diskussionswürdig. Gleichzeitig ist `Sprechen` im Verhältnis zu den vielfältigen Formen und Funktionen der Lautgebung - sei dies Gesang oder Skansion, Rezitation oder Deklamation - ungenau formuliert. Die Redeweise vom 'Sprecher des Gedichts könnte zudem suggerieren, dass Sprache in der Lyrik in einem gewöhnlichen Kommunikationszusammenhang verwendet werde. Inwiefern aber für Lyrik (gegebenenfalls spezifisch literarische) Kommunikationsnormen in Anschlag gebracht werden können, ist interpretationstheoretisch nicht unumstritten. ${ }^{3}$ Hier berührt die Frage nach der `Äußerungsinstanz` neben der Modalität auch kommunikationstheoretische Fragen des lyrischen Sprechens, Schreibens oder auch Singens.

〉Gedicht`? Die Frage »Wer spricht das Gedicht?«, die Borkowski und Winko als Titel ihres lyrikologischen Aufsatzes von 2011 setzten und die wir hier in leicht abgewandelter Form übernommen haben, ist eine Anspielung auf Wolfgang Kaysers Aufsatz »Wer erzählt den Roman?« (1957). Vermutlich um des Reimes willen wählen schon Borkowski und Winko den Gattungsnamen `Gedicht «, obwohl hier mit gutem Recht von `Lyrikı die Rede sein könnte. Wie im vorliegenden Band, so geht es auch bei Borkowski und Winko nicht etwa um `dramatische Gedichte`,

2 Mehrere Beiträge im vorliegenden Band sind das Ergebnis eines vom Schweizerischen Nationalfonds geförderten Workshops, der im Oktober 2015 an der Universität Freiburg (Schweiz) zur Frage »Wer spricht im Gedicht?« stattfand. Die überarbeiteten Beiträge werden hier in einen systematischen Zusammenhang gestellt und um ausgewählte zusätzliche Aufsätze ergänzt.

3 Vgl. dazu z. B. den Beitrag von Rüdiger Zymner in diesem Band. 
sondern schlicht um Lyrik. Es ist zwar denkbar, dass in Zukunft die Abgrenzung von Lyrik und Gedicht auch in der deutschen Sprache wieder stärker gemacht wird (vgl. schon Lamping 1989/2000, zur angloamerikanischen Kritik vgl. Jackson und Prins 2014, zur Gegenposition vgl. Culler 2015, 82-83); jedoch lässt sich der Gattungsbegriff >Lyrik» bei der Frage »Wer spricht im Gedicht? « schwerlich ausschließen. Es ist sogar ratsam, die Wortwahl hier explizit auszuweisen, bedenkt man Lampings (1989/2000, 19-38) Vorschlag, präzisierend eine trennscharfe Definition des >lyrischen Gedichts` vorzunehmen, oder Burdorfs (1995/2015, 20) entgegengesetzten Ratschlag, auf eine Differenzierung von `Lyrikı und `Gedicht zu verzichten.

Unter dem Blickwinkel einer systematischen Theorie der Lyrik wird sich schließlich sogar die scheinbar harmlose Präposition ıim`als fragwürdig erweisen, scheint sie doch die Suche auf eine Instanz innerhalb des Textes festzulegen, obwohl es für manche Gedichtgenres denkbar ist, die Antwort außerhalb des Textes, insbesondere in der Person des empirischen Autors oder des >Performers zu suchen.

Ein knapper Überblick über einige lyrikologische Vorschläge zur Beantwortung der Ausgangsfrage dieses Bandes kann einen Eindruck von der Lebendigkeit, aber auch von der Heterogenität der aktuellen Debatten geben, die bislang in den verschiedenen Philologien in unterschiedlichen Sprachen separat geführt wurden und sich momentan international zu einem Forschungsdiskurs zu vernetzen beginnen. Problematisch auf den interphilologischen Austausch wirken sich dabei die heterogenen Praktiken des Benennens von lyrischen >Äußerungsinstanzen in den unterschiedlichen Sprachen, aber auch in den verschiedenen Wissenschaftstraditionen aus. Außerhalb des deutschsprachigen Raums hört man als Antwort auf die Eingangsfrage »Wer spricht im Gedicht? « häufig Stich-

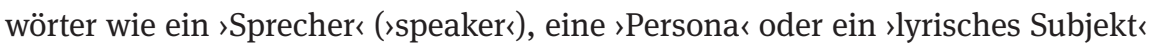
(১moi lyrique` bzw., häufiger verwendet, `sujet lyrique`). Blickt man demgegenüber in die Praxis der germanistischen Gedichtanalyse, so stellt man fest, dass die Frage »Wer spricht im Gedicht? « immer noch sehr häufig mit »das lyrische Ich» beantwortet wird. Die ungebrochene Popularität dieser Kategorie ist zunächst erstaunlich angesichts der widersprüchlichen Begriffsgeschichte (für eine Übersicht vgl. Müller 2011). Während die Literaturwissenschaftlerin Margarete Susman (1910) den Begriff prägte, um für moderne Lyrik deutlich zwischen dem empirischen Autor und der Allgemeingültigkeit der Aussagen des durch ihn geschaffenen lyrischen Ichs zu unterscheiden, identifizierte Käte Hamburger (1977, 217) das lyrische Ich im Gegenteil mit dem »realen Aussagesubjekt « des Textes. Wiederum anders verstand Gottfried Benn den Begriff des lyrischen Ichs, das er mit einer nur vage beschriebenen dichterischen Inspirationsquelle, in seinen Worten der »Inkarnation alles dessen, was an lyrischem Fluidum in dem Gedichte produ- 
zierenden Autor lebt« (Benn 1989, 551), identifizierte. Fehlt noch die Erwähnung jüngerer >Rettungen` des lyrischen Ichs, die vorschlagen, den Begriff auf die `Äußerungsinstanz in denjenigen Gedichten einzugrenzen, in denen keine fiktive Figur sspricht` (also für Gedichte, die nicht offenkundig zur Rollenlyrik zählen, wie etwa Clemens Brentanos »Der Spinnerin Lied«), und in denen offenbleibt, ob die Äußerungsinstanz des Gedichts mit dem empirischen Autor identisch ist oder nicht. Das lyrische Ich wäre demnach eine »Leerdeixis«, zu deren Füllung sich Rezipienten den empirischen Autor, eine fiktive Figur oder gar sich selbst vorstellen können (vgl. Martínez 2002; Fricke und Stocker 2000 unter Bezug auf Spinner 1975).

Zweifel an der »Nützlichkeit « des Begriffs 〉lyrisches Ich`als einem »unverwüstliche[n] Wiedergänger« (Killy 1972, 4) wurden dagegen vor dem Hintergrund der widersprüchlichen Begriffsprägungen geäußert (vgl. Burdorf 1995/2015, 191-194; auch Borkowski und Winko 2011, 43-44). Borkowski und Winko verwenden in ihrem Modell zur Beschreibung von Äußerungsinstanzen in Lyrik stattdessen den Ausdruck >Sprecher (Borkowski und Winko 2011, 64), ähnlich wie dies auch die narratologisch informierte Lyriktheorie tut (vgl.z. B. Hühn und Kiefer 2005, 8-9). ${ }^{4}$ Aufgrund des verwirrenden und widersprüchlichen Gebrauchs empfiehlt auch Burdorf, den Begriff des lyrischen Ichs »fallenzulassen und das Problem mit einer neuen Begrifflichkeit anzugehen« (Burdorf 1995/2015, 192), indem fortan mit Nägele (1980, 62) von einem sartikulierten Ich`(Burdorf 1995/2015, 194) gesprochen werden solle. Er führt darüber hinaus zusätzlich den Begriff des >Textsubjekts` ein: »Es strukturiert die Perspektive des Gedichts und setzt das Ich, ohne mit ihm identisch zu sein. Daher ist es auch als der textinterne Ausgangspunkt der anderen Personen anzusehen; es ist selbstverständlich auch in Gedichten vorhanden, in denen kein Ich zur Sprache kommt.« (Burdorf 1995/2015, 195)

Es ist nicht das Ziel dieser Einleitung, die begründete Skepsis und Vorsicht gegenüber dem Begriff des >lyrischen Ichs` bzw. die Vorschläge für seinen kontrollierten Gebrauch oder für alternative Begriffsbildungen aufzuarbeiten. Das ist andernorts bereits sehr gründlich geschehen (vgl. Borkowski und Winko 2011; Müller 2011; Martínez 2002). An der Begriffsgeschichte des `lyrischen Ichs` zeigt sich aber beispielhaft, dass die mit der Begriffsbildung verbundenen theoretischen Fragen auch praktische Relevanz für den analytischen und interpretativen Umgang mit Lyrik haben - Fragen, die nach unserem Dafürhalten insbesondere der systematischen Reflexion über `Äußerungsinstanzen` in der Lyrik bedürfen.

Im Zentrum dieser Fragen steht die Konstitution und damit die Personalität von lyrischen `Äußerungsinstanzen`, und zwar zum einen im Hinblick auf die

4 Vgl. dazu z. B. den Beitrag von Evelyn Dueck in diesem Band. 
Relation zwischen `Äußerungsinstanz^ und Urheber bzw. Kontext des lyrischen Gebildes, zum anderen im Hinblick auf die `Äußerungssituation`selbst. Diese beiden Aspekte wollen wir hier etwas ausführlicher thematisieren, denn sie zeigen Grundlinien auf, an denen sich unseres Erachtens eine Reform der lyrikologischen Begriffssprache orientieren könnte. Unsere nun zu erläuternden fiktionstheoretischen und modalitätsspezifischen Prämissen werden dazu dienen, den Begriff des `lyrischen Ichs`, aber auch alternative Begriffsbildungen wie etwa ১Sprecher oder >artikuliertes Ich ' und dessen Komplement, das >Textsubjekt $\iota,{ }^{5}$ mithilfe des Begriffspaars `Adressant - >Adressat neu zu betrachten. Wir hoffen, dass diese neue Terminologie der Lyrikologie durch ihre fiktions- und modalitätsspezifische Neutralität neue Impulse gibt, indem sie den Blick für das komplexe Zusammenspiel von Faktoren schärft, die bei der Analyse von `Äußerungsinstanzen ` in Lyrik zu berücksichtigen sind, und damit hoffentlich auch zur oben erwähnten besseren Vernetzung des internationalen Forschungsdiskurses beitragen wird.

\section{Fiktionalität}

Die wechselvolle Begriffsgeschichte des `lyrischen Ichs ist auch eine unterschiedlicher Konzeptionen der Fiktionalität bzw. Nicht-Fiktionalität von Lyrik. Auf die konträren Positionen Hamburgers und Susmans wurde oben bereits hingewiesen. Hamburgers Annahme, lyrische Gebilde seien stets nicht-fiktional, wird heute in der Lyrikologie kaum noch vertreten. Panfiktionalistische Positionen finden sich demgegenüber noch häufiger. So haben zum Beispiel Werner Wolf und neuerdings Klaus W. Hempfer für die Auffassung plädiert, dass Lyrik stets fiktional sei. Während Wolf diese Annahme eher en passant äußert (vgl. Wolf 2005, 23), begründet Klaus W. Hempfer diese Auffassung ausführlicher mit Bezug auf die sprechakttheoretische Fiktionstheorie von John Austin und beschreibt sie genauer als »Performativitätsfiktion« (vgl. Hempfer 2014, 32, 34). Hempfer geht davon aus, dass die Fingierung einer Simultaneität von Sprech- und besprochener Situation, die prototypisch lyrische Gebilde ihm zufolge aufweisen, deren Fiktionalität kon-

\footnotetext{
5 Auf den Begriff des `Textsubjekts möchten wir verzichten, weil er das hochgradig umstrittene Konzept des simpliziten Autors aus der Erzähltheorie in die Lyrikologie importiert und dieser damit einen schillernden Begriff hinzufügt, dessen Nutzen auch in der Narratologie noch keineswegs als erwiesen und dessen Gebrauch nicht als allgemein akzeptiert angesehen werden kann. Mit diesem Begriff wird darüber hinaus ein Modell lyrischer Kommunikation impliziert, das, wie oben bereits angemerkt worden ist, für die Lyrikologie erst noch zu diskutieren wäre. Vgl. kritisch zum simpliziten Autor` Kindt und Müller 2006 sowie 2011.
} 
stituiert. Allerdings machen auch andere Textsorten wie beispielsweise Reportagen und Briefe von einer solchen fingierten Simultaneitätsrelation Gebrauch, ohne dass diese Textsorten deswegen als fiktional angesehen würden. Austins Fiktionstheorie ist unter anderem wegen dieser mangelnden Trennschärfe gegenüber Formen nicht-fiktionaler Sprachverwendung in der neueren Fiktionstheorie mit guten Gründen kritisiert worden (für eine ausführliche Auseinandersetzung vgl. Gertken und Köppe 2009, 242-244).

In der neueren Fiktionstheorie wie auch in der Lyrikologie wird dagegen mittlerweile überwiegend die Auffassung vertreten, dass Lyrik und Fiktion unabhängig voneinander zu bestimmende Konzepte seien (für die Fiktionstheorie vgl. exemplarisch Zipfel 2011, für die Lyriktheorie Lamping 2000, 102-110, zu ihrer grundsätzlichen Unabhängigkeit vgl. bereits Anderegg 1985/2000). Dieser Auffassung schließen sich die Herausgeber dieses Bandes an. Aus dieser Sichtweise ergibt sich, dass nicht pauschal, sondern nur fallweise geklärt werden kann, ob es sich bei der `Äußerungsinstanzı eines lyrischen Gebildes um eine fiktive oder eine reale Instanz handelt und dass eine systematische Terminologie daher im Hinblick auf eine grundsätzlich unterstellte (Nicht-)Fiktionalität der Lyrik neutral gehalten sein sollte. Konsequenterweise hat beispielsweise Rüdiger Zymner in seiner Typologie zwischen autorfiktionalen, personafiktionalen, autorfaktualen und personafaktualen Gedichten unterschieden (Zymner 2009, 10-12). Diese Typologie macht deutlich, welche Kontexte bei der Analyse von lyrischen >Äußerungsinstanzen $` \mathrm{zu}$ berücksichtigen sind, welche Schlüsse vom Gebilde selbst auf die Wirklichkeit also sinnvoll sind. Da die Debatte über das ılyrische Ich zum Teil so geführt wurde, als seien Lyrik und Fiktion voneinander abhängige Größen und die Begriffsprägung >lyrisches Ich`daher immer schon fiktionstheoretisch imprägniert, und da entsprechende Begriffsprägungen leider weiter in Gebrauch sind (vgl. den Überblick bei Borkowski und Winko 2011, 53-54), ist es - um hier nur diesen einen zentralen Kritikpunkt an dem Begriff `lyrisches Ich` noch einmal aufzugreifen - aus unserer Sicht sinnvoll, einen Begriffsnamen zu wählen, der diese problematischen Konnotationen vermeidet. Darüber hinaus sind zwei weitere Aspekte der Fiktionsfrage von hoher lyrikologischer Relevanz:

Zum einen ist es derjenige, ob die Fiktionalität eines lyrischen Gebildes durch das Vorhandensein einer fiktiven >Äußerungsinstanzı konstituiert wird. Diese Frage stellt sich analog zu der in der narratologischen Fiktionstheorie, wo häufig davon ausgegangen wird, dass die Fiktionalität einer Erzählung durch das Vorhandensein eines fiktiven Erzählers konstituiert wird (vgl. Petzold 2012). Dieser Position ist $\mathrm{zu}$ Recht vorgeworfen worden, dass sie Ursache und Wirkung verwechsele: Nicht die Existenz eines fiktiven Erzählers konstituiert die Fiktionalität eines Erzähltextes, sondern aus der Erkenntnis, dass ein Erzähltext fiktional ist, lässt sich, wenn ein Erzähler erkennbar ist, die Schlussfolgerung ableiten, 
dass dieser nicht ohne Weiteres mit dem Autor oder einer anderen nicht-fiktiven Person identifiziert werden darf (vgl. Gertken und Köppe 2009, 237-238). Wenn aber das Vorhandensein einer fiktiven Erzähl- oder Äußerungsinstanz für die Fiktionalität oder Nicht-Fiktionalität eines Sprachgebildes gar nicht notwendig ist, so bedeutet das auch, dass nicht jedes fiktionale Sprachgebilde automatisch eine fiktive Äußerungs- oder Erzählinstanz aufweisen muss (vgl. Köppe und Stühring 2011). Daraus lässt sich die Schlussfolgerung ableiten, dass nicht jedes lyrische Gebilde eine Äußerungsinstanz aufweist und dass dies auch terminologisch mitbedacht werden sollte.

Zum anderen sind solche Vorschläge wie derjenige von Harald Fricke und Peter Stocker zu bedenken, die angemerkt haben, dass die Relation zwischen der Äußerungsinstanz in einem lyrischen Gebilde und seinem Urheber bzw. seines Kontextes keineswegs immer klar sein muss (vgl. Fricke und Stocker 2000). Im Gegenteil ist es oft so, dass die Fiktionalität bzw. Nicht-Fiktionalität eines lyrischen Gebildes nicht genau ermittelt werden kann (vgl. Zipfel 2011, 166) und damit unklar bleibt, welche Kontexte für die Konstitution (und Analyse) der Äußerungsinstanz einschlägig sind. Fricke und Stocker schlagen vor, von einem >Lyrischen Ich، stets und nur in genau diesen Fällen zu reden. Auch wenn dieser Explikationsvorschlag sich unseres Erachtens nicht durchgesetzt hat, so sollte dieser gerade für lyrische Gebilde häufig auftretende Fall doch terminologische Berücksichtigung finden - gegebenenfalls eben unter einem anderen Begriffsnamen.

Die Kritiker der Begriffsprägung >lyrisches Ich`schlagen statt seiner eine mündlichkeitsmetaphorische Begrifflichkeit vor, wenn sie - wie Borkowski und Winko oder Hühn und Kiefer - >Sprecher` oder - wie Burdorf - >artikuliertes Ich verwenden. Diese Begriffsprägungen verdanken sich zum einen dem Terminologietransfer aus der Narratologie, den die Lyriktheorie in den letzten Jahren erlebt hat (vgl. Müller-Zettelmann 2002, Schönert, Hühn und Stein 2007), zum anderen aber der Vorstellung, dass das eigentlich angemessene Medium der Lyrik die gesprochene Sprache sei (vgl. Burdorf 2015, 32). Allerdings transportiert auch diese Mündlichkeitsmetaphorik einige problematische Konnotationen, die unseres Erachtens vermieden werden sollten.

\section{Modalität}

Die Herausforderungen der >Modalität in der Lyrik lassen sich annäherungsweise anhand eines Beispiels darstellen: Hugo Balls Lautgedicht »Karawane» bzw. »Zug der Elefanten« (vgl. Ball 2007, 68) ist vermutlich am bekanntesten in 
einer - von Hugo Ball übrigens nicht autorisierten - Fassung (vgl. Ball 2007, 220), die sich nicht nur durch eine von üblicher Semantik abweichende Lautbildung auszeichnet, sondern in der auch die Verszeilen zusätzlich durch die Verwendung unterschiedlicher Schriftarten markiert sind. Eine Beschreibung dieser Fassung mit einer bloßen Wiedergabe der assoziationsreichen, aber unbekannten Laute »jolifanto bambla [...] « wäre in dieser Hinsicht unbefriedigend. Auch die Collage unterschiedlicher Schriftarten trägt zur Bedeutungsgenerierung dieser Gedichtfassung bei. Die graphische Repräsentation als Zusammenspiel von verbalen, nonverbalen und paraverbalen Ressourcen wie Schriftarten, Anordnung von Verszeilen etc. könnte zum Beispiel einen Eindruck von Vielstimmigkeit oder eines unkonventionellen Ichs erzeugen (muss es aber nicht). Es wäre problematisch, aufgrund der besonderen drucktechnischen Gestaltung bereits von der Verwendung sdistinkt wahrgenommener Medien ( vgl. Rajewsky 2002) auszugehen. Mit van Leeuwen und Kress (2017, 20-22) lässt sich dies besser als ein >multimodales Gebilde betrachten, das auf unterschiedliche semiotische Ressourcen (verbal, nonverbal und paraverbal) rekurriert. Gleichzeitig lässt sich diese Textfassung nur unvollständig als Aussage eines Sprechers oder gegebenenfalls mehrerer Sprecher beschreiben, ebenso problematisch scheint es, diese Laute einem Ich oder gar einem Wir zuzuschreiben. Auch wenn der Eindruck der Präsenz einer wie auch immer gearteten Äußerungsinstanz evoziert wird, sind von solchen Instanzen im Text selbst keine konkreten Spuren nachweisbar. Dabei hatte das Gedicht sehr wohl Sprecher: Die erste Veröffentlichung erlebte das Lautgedicht als - wie man heute sagen würde - >Performance`, von der wir keine audiovisuellen Aufnahmen, aber immerhin Beschreibungen und Fotografien von Hugo Ball besitzen, wie er als "magischer Bischof « seine Lautgedichte zelebrierte (vgl. Ball 2007, 216- 220). Auch eine solche phonische Version wäre im Hinblick auf die jeweilige Performance zu betrachten, die nicht auf einen mündlichen Text reduzierbar ist, sondern Bedeutung aus komplexen Parametern wie Ort der Darbietung, nonverbale Zeichen, Lautstärke, Rhythmus etc. (vgl. hierzu allg. Novak 2011, 75-144) bezieht.

>Modalität ist also nicht gleichbedeutend mit dem grammatischen Modus oder Genettes Differenzierung von dramatischem und narrativem Äußerungsmodus, zwischen denen übrigens Genette gerade für die Lyrik keinen Raum mehr sah (vgl. Genette 1979, 66 f.). `Modalitätı, wie es hier verstanden wird, erfordert ein differenziertes Begriffsrepertoire zur Analyse von phonischen oder graphischen Qualitäten lyrischer Sprachgebilde (vgl. in dieser Hinsicht die Hinweise im Glossar) sowie grundsätzliche begriffliche Konsequenzen allgemeinerer Art. Die begrifflichen Konsequenzen betreffen zum Beispiel den Begriffsnamen 'Sprecher`, der als möglicher Ersetzungsvorschlag für das `lyrische Ich` bisweilen auftaucht (vgl. Borkowski und Winko 2011). Die Vorteile dieses Begriffsnamens sind nicht zu übersehen: `Sprecher lässt sich nicht nur an den in angloamerika- 
nischen Studien verbreiteten Begriff des >speaker ‘ anschließen, sondern etabliert zugleich eine Parallele zum Begriff des `Erzählers` in der Narratologie. Wie tragfähig ist aber die Analogie zum `Erzähler` in der Narratologie (vgl. hierzu affirmativ Hühn und Schönert 2007)? In Anlehnung an die Narratologie könnte man ১Sprecher als wortwörtliche Antwort auf die Genette'sche Frage »qui parle?« im Gedicht auffassen. Genette hat in der Narratologie diese Frage auf die Ermittlung der Erzählstimme, also des Erzählers, ausgerichtet, dies in Abgrenzung der den Modus des Erzählens betreffenden Frage der Fokalisierung von »qui voit? «. ${ }^{6}$ Im Falle von Lyrik ist aber unseres Erachtens im Hinblick auf die Frage »qui parle?« nicht nur die (1) referenzielle Klärung relevant, sondern es ergeben sich zudem die Fragen der (2) phonischen oder graphischen Repräsentation von lyrischen Sprachgebilden (Modalität) sowie (3) nach den genre- oder kulturspezifisch etablierten Konventionen solcher Realisierungen.

(1) Die Referenzialität ist bereits gründlich im Zusammenhang der Personalität von Lyrik angesprochen worden. Wenn sie hier nochmals zum Zuge kommt, dann nur aus dem Grund, dass es auch darum geht, welche Arten von `Sprecher `Äußerungsinstanzen ๖ bei lyrischen Sprachgebilden in Frage kommen könnten. Wie Borkowski und Winko (2011) in ihrer Typologie von lyrischen Sprechhaltungen dargelegt haben, kann die Frage »wer spricht? « zu drei unterschiedlichen referenziellen Befunden führen, nämlich dass ein Gedicht `sprecherlos` ist (wenn zum Beispiel bei manchen Dinggedichten keine greifbare Äußerungsinstanz angenommen werden muss), dass ein textinterner Sprecher oder ein textexterner Sprecher vorliegt (vgl. Borkowski und Winko 2011, 75 f.). Aus der Sicht der Narratologie dürfte vor allem die Zweiteilung des `Sprechers` in einen `textinternen und stextexternen Sprechers` bei Borkowski und Winko lässt sich mit verschiedenen Praktiken im Umgang mit Lyrik begründen. Etwa mit der verbreiteten Praxis, bestimmte Gedichte laut zu lesen. Hier liegt auch eine wichtige Differenz zum Erzählen vor. Zwar werden auch Erzählungen vorgelesen und können, auch wenn die Konvention des Lesens als `Subvokalisation` vorherrscht, einen textexternen Sprecher aufweisen. Man denke an Autorenlesungen, abendliches Vorlesen für Kinder oder an Hörbücher. Gegenüber diesen Beispielen steht aber die verbreitete Forderung, Lyrik müsse laut gelesen oder rezitiert werden. ${ }^{7}$ Ebenso ist es breit akzeptiert,

6 Mehrfach wurde kritisiert, dass die Frage »qui voit? « für die vielfältigen Aspekte der Fokalisierung zu einseitig formuliert ist, nicht zuletzt Genette (1994, 235) hat die Visualität der Formulierung bedauert (differenzierter zu dieser Frage vgl. z. B. Schmid 2005, 115-153).

7 Vgl. hierzu kritisch Burdorf 2015, 32. Tatsächlich eignen sich Gedichte nicht im selben Maße für eine lautliche Realisierung. So bedürfen zum Beispiel Apollinaires Calligrammes als Figuren- 
dass sich Rezipienten mit dem Ich identifizieren dürfen, dies legen nicht zuletzt verschiedene theoretische Ansätze zum ılyrischen Ich` nahe (vgl. Schlaffer 2012, Spinner 1975). Man kann dies als Hinweis auf eine potenzielle Offenheit der Referenz des Sprechers zumindest bei bestimmten lyrischen Genres betrachten. Problematisch ist allerdings, dass ıSprecher unter diesen Bedingungen mehrdeutig wird. Es kann einen tatsächlichen textexternen Sprecher und einen postulierten textinternen Sprecher bezeichnen, unter Umständen sogar beides, aber auf der Grundlage unterschiedlicher Kriterien, wie das Hugo-Ball-Beispiel gezeigt hat. Der begriffliche Rekurs auf ein >Ich`, wie man ihn im ১lyrischen Ich oder >artikulierten Ich`antrifft, weist gegenüber dem >Sprecher « eine systematische Offenheit der Deixis auf (`Äußerungsinstanz` ist immer, wer inner- oder außerhalb des Texts >ich`sagt), löst aber - wenn es mit einer undifferenzierten Vorstellung von ıSprechen oder >Artikulation` verbunden wird - nicht die grundlegenden Probleme der Differenzierung von phonischer und graphischer Repräsentation lyrischer Sprachgebilde. `Textinternes Sprechen` ist also als metaphorische Ausweitung von lautlicher Realisierung auf Schrift zu betrachten.

(2) Aus dem Vorangegangenen wird deutlich, dass stextinternes externes Sprechen spiele für eigentliches `Sprechen`, etwa bei einer Lyriklesung, beim Rezitieren eines Gedichts oder auch beim inneren subvokalen Verlautlichen eines Gedichts. Textintern haben wir es üblicherweise mit einem Text zu tun, über dessen potenzielle phonische Realisierung wenig bekannt ist (es sei denn, das Gedicht ist als Figurenrede durch einen Erzähltext eingerahmt). Mit `Sprechen` werden unterschiedliche phonische und graphische Realisierungsmöglichkeiten von Gedichten auf eine bestimmte Form der Darbietung (`sprechen`) eingeschränkt; dies unter Ausschluss der möglicherweise komplexen graphischen Repräsentation und unter Ausschluss zahlreicher Parameter, die eine phonische Realisierung in Abweichung von neutralem Sprechen charakterisieren können. Paradoxerweise wird dieses `Sprechen`, das Mündlichkeit suggeriert, hauptsächlich an graphisch repräsentierten Sprachgebilden beschrieben. Der kategoriale Unterschied wird auch dadurch deutlich, dass das »ich « und »du« textintern ihre potenziellen textexternen Referenten überleben - dies ist etwa in den letzten Zeilen von Shakespeares Sonett Nummer $18 \mathrm{zu}$ finden: „So long as men can breathe, or eyes can see, / So long lives this, and this gives life to thee«. Dichter haben also schon lange die Differenz von >textexternem ‘ und >textinternem Sprechen $>$ zu nutzen gewusst, es ist aber zweifelhaft, ob die Literaturwissenschaft von einer fehlenden Differen-

gedichte einer Rezeption in der graphischen Realisierung. In vielen Fällen hat lautes Lesen außerdem einen komischen Effekt (z. B. Handkes » Aufstellung des 1. FC Nürnberg vom 27. 1. 1968«). 
zierung von tatsächlichem Sprechen und seiner metaphorischen Ausweitung auf Schriftlichkeit profitiert. Eine medienunabhängige Benennung der Äußerungsinstanz könnte nicht zuletzt dazu beitragen, den phonischen Versionen von lyrischen Gedichten, die aufgrund der Möglichkeit audiovisueller Speicherung von Dichterlesungen und -performances für Analysen zunehmend zur Verfügung stehen, eine eigene Geltung einzuräumen (vgl. Novak 2011, 19-20, 62). Ebenso wäre es wünschenswert, den graphischen Repräsentationsweisen mit größerer Präzision gerecht zu werden.

(3) Schließlich möchten wir generische und kulturhistorische Argumente für eine Ausdifferenzierung von Sprechhaltungen erwähnen. Man trifft bisweilen auf die Ansicht, dass >wahre ‘ Lyrik für sich selbst stehen kann und auch in der Performance keiner zusätzlichen Effekte bedürfe (zu dieser Auffassung vgl. Novak 2011, 26-27). In diesem Sinne ostentativ zurückhaltende Darbietungsweisen lassen sich gut als >Sprechen` verstehen. Allerdings ist Lyrik in der Geschichte ihrer Poetik auch mit intensiveren phonischen Realisierungen wie Gesang oder Anrufung assoziiert worden. Aber schon gegenüber der >Deklamation` (vgl.z. B. Vischer 1857, §858) erscheint `Sprechen` als eine historisch beschränkte generische Konvention. In dieser Hinsicht betreffen Fragen der >Modalitätı schließlich die Gattungsdefinition. Nimmt man `Sprechen ‘ in einem strengen Sinne, dann gehören Genres mit anderen phonischen Realisierungsweisen wie `slam poetry‘, Lieder und Songs nicht mehr in das Gattungsspektrum der Lyrik.

\section{Zum Begriff des sAdressanten}

Schwierigkeiten, theoretische und methodische Probleme beim literaturwissenschaftlichen Umgang mit >der Äußerungsinstanz` hängen nun insbesondere damit zusammen, dass bislang häufig davon abgesehen wird, dass man es bei Lyrik zunächst und zuerst mit Sprachzeichengebilden zu tun bekommt. Lyrik - das sind (besondere) schriftlich fixierte oder verlautlichte Sprachzeichengebilde (also Zeichengebilde, die Sprache fixieren oder repräsentieren oder manifestieren). Dieser Umstand wäre nun allerdings deutlich und in aller Konsequenz in den Mittelpunkt der Theoriebildung zu rücken. Dann könnte man vielleicht besser sehen, dass auch diese vermeintliche `Äußerungsinstanz` selbst zunächst einmal nichts anderes als ein Sprachzeichengebilde ist, oder besser: sich erstens aus Sprachzeichen des lyrischen Sprachzeichengebildes konstituiert, welche zweitens wiederum ein kognitives Konzept - eine vage Vorstellung - einer womöglich sogar anthropomorphen >Äußerungsinstanz` in der Rezeption triggern. Die Sprachzeichen selbst sind dabei natürlich nicht anthropomorph, sondern eben 
arbiträre und in gewisser Weise abstrakte Schriftzeichen oder arbiträre und in gewisser Weise abstrakte Laute; und die getriggerten kognitiven Konzepte sind keine eigenständigen Subjekte, die es irgendwo unabhängig von den Vorstellungen des Rezipienten gäbe, sondern kognitive Effekte, die von den Sprachzeichen und den durch sie mehr oder weniger konventionellen Bedeutungen bestimmt werden. Weder dieser `Äußerungsinstanz` als bloßes Sprachzeichen noch dem hierdurch getriggerten kognitiven Konzept kann man eigene, selbständige Tätigkeiten oder ein eigenes, selbständiges Bewusstsein und schon ganz und gar nicht die Identität mit dem Autor zuschreiben, weil der Autor ein Mensch aus Fleisch und Blut ist, das Sprachzeichengebilde und der hierdurch getriggerte kognitive Effekt aber ganz sicher nicht. Kognitive Effekte sind keine eigenständigen Entitäten, und Sprachzeichen tun nichts (sondern sie werden allenfalls als Anzeichen für etwas anderes, Abbildungen von etwas anderem oder Symbole für etwas anderes gedeutet); kognitive Effekte und Sprachzeichen selbst haben kein Bewusstsein, und der Autor ist etwas ganz anderes als die Sprachzeichen, die er schreibt oder sagt/singt, und deren kognitive Effekte - auch wenn diese Sprachzeichen als kognitiver Effekt in manchen Fällen auf den Autor verweisen mögen und so die Einschätzung unterstützen können, dass sich der Autor selbst über tatsächliche (und nicht nur fiktive) Sachverhalte mit diesen Sprachzeichen äußere. Sprachzeichengebilde sprechen auch nicht - jedenfalls tun dies graphisch manifeste lyrische Sprachzeichengebilde nicht -, und phonisch manifeste lyrische Sprachzeichengebilde werden gesprochen oder gesungen, sie selbst können sich gewissermaßen in keinem Fall (laut) mitteilen.

Lyrikologische Forschungen zur `Äußerungsinstanz« sollten also zunächst einmal die alte zeichentheoretische Regel berücksichtigen, dass ein Zeichen nicht die Sache selbst ist, die es bezeichnet; dass Schrift lautlos ist und bleibt, Laute aber von Lebewesen hervorgebracht werden müssen und alle Schriftstücke von einem Autor oder einer Autorin (fallweise auch von einem intermediären Schreiber oder einer Schreiberin) geschrieben worden sind und immer jetzt (im jeweiligen Jetzt eines Lesers aus Fleisch und Blut) gelesen werden.

Um die semiotischen Sachverhalte von allen hiermit assoziierten Weiterungen trennen zu können, mag es sinnvoll sein, die betroffenen semiotischen Fälle begrifflich deutlich voneinander zu unterscheiden und in Abhebung von einer umstrittenen Begriffsverwendung auch zu benennen. Zu diesem Zweck schlagen wir vor, erstens den Begriffsnamen >Adressant $\mathrm{zu}$ verwenden, weil er im Unterschied zu der bisher herangezogenen Terminologie (vor allem >lyrisches Ich`, `Sprecher`, aber auch `Äußerungsinstanz`) nicht oder wenigstens nicht leicht mit unzutreffenden oder sogar irreführenden Vorstellungen (vor allem solchen von einem eigenständigen Subjekt, das beispielsweise ıspricht‘, wo tatsächlich nur Schriftzeichen vorliegen) verknüpft werden kann; und wir schlagen zweitens vor, 
mit dem Ausdruck >Adressant « die Markierung eines pragmatischen Ausgangspunktes des lyrischen Sprachzeichengebildes in dem Sprachzeichengebilde selbst und mit den Mitteln des Sprachzeichengebildes zu bezeichnen: »in dem Sprachzeichengebilde« meint die Konstellation oder auch Sequenzialisierung der Sprachzeichen des Sprachzeichengebildes als (abgegrenzte, abgeschlossene) Einheit; »mit den Mitteln des Sprachzeichengebildes « meint die durch die Sprachzeichen bewerkstelligten grammatischen, stilistischen, rhetorischen, metrischen usw. Verfahren.

Als »Adressant« wird also keine >Person` oder >Instanz« und kein `Subjekt» bezeichnet, sondern zunächst lediglich eine spezifische Zeichenstruktur des lyrischen Sprachzeichengebildes selbst - ein semiotischer, hier auf Konstellation, Sequenzialisierung und Verfahren von Sprachzeichen beruhender Sachverhalt. Der >Adressant als Zeichenstruktur, als semiotischer Sachverhalt ist dabei zu unterscheiden von dem tatsächlichen praktischen Ausgangspunkt des Sprachzeichengebildes außerhalb des Sprachzeichengebildes: Dies ist in der Regel der Autor. Der >Adressant kann als eine fiktive lyrische Persona gestaltet sein (etwa in einem sogenannten >Rollengedicht`); der >Adressant kann direkt auf den faktischen Autor verweisen oder schließlich auch nicht entscheidbar (adressantenneutral) im Hinblick auf Fiktivität oder Faktizität bleiben. Lyrische Sprachzeichengebilde können auch ganz ohne Adressanten auskommen (z. B. Konkrete Lyrik, Einwortgedichte, Carmina figurata, lyrische Schriftzeichen, Schriftzeichengebilde, Redezeichen etc., aber auch manche Dinggedichte wie C. F. Meyers »Der römische Brunnen « oder Rimbauds »Marine«, in denen beispielsweise keine Personalpronomen wie »Ich« oder »Wir« und diesen Personalpronomen zugeordnete verba dicendi, agendi oder sentiendi vorkommen und auch an stilistischen Besonderheiten oder an einer besonderen Perspektivgestaltung kein pragmatischer Ausgangspunkt des Sprachzeichengebildes erkennbar wird); sie sind dann adressantenfrei.

Das semiotische Gegenstück zum >Adressanten ` ist der >Adressat ‘. Mit diesem Begriffsnamen verbinden wir die Markierung einer pragmatischen Zielorientierung des lyrischen Sprachzeichengebildes in dem Sprachzeichengebilde selbst und mit den Mitteln des Sprachzeichengebildes. Als >Adressat` wird also entsprechend zu dem Konzept des >Adressanten « keine >Person « oder `Instanz « bezeichnet, sondern ebenfalls eine spezifische Zeichenstruktur des lyrischen Sprachzeichengebildes selbst. Der >Adressat` als Zeichenstruktur ist von einem tatsächlichen Empfänger des lyrischen Sprachzeichengebildes (dem Leser oder Hörer) als seiner praktischen Zielorientierung zu unterscheiden. Der Adressat kann als eine fiktive Figur gestaltet sein; der Adressat kann auf einen faktischen Empfänger verweisen oder schließlich auch - adressatenneutral - nicht entscheidbar neutral im Hinblick auf Fiktivität oder Faktizität bleiben. Lyrische Sprachzeichengebilde können 
auch ganz ohne Adressaten auskommen (z. B. Konkrete Lyrik, Einwortgedichte, Carmina figurata, lyrische Schriftzeichenzeichen, Schriftzeichengebilde, Redezeichen etc.; aber auch Dinggedichte); sie sind dann adressatenfrei.

\section{Beiträge in diesem Band}

Der vorliegende Band versammelt eine Reihe von Studien, die die Frage der Adressantenkonstitution in der Lyrik aus unterschiedlichen Perspektiven historisch-fallorientiert, wissenschaftsgeschichtlich oder systematisch angehen.

Die Beiträge sind im Folgenden in vier Sektionen gegliedert. Die erste Sektion widmet sich begriffsgeschichtlichen und systematischen Überlegungen zur Beschreibung von »Adressantenformen in der Lyrik«. In der zweiten Sektion werden unter dem Stichwort »Fiktionalität« verschiedene interpretationstheoretische Vorannahmen behandelt. So stellt sich die Frage, in welchem Ausmaß Adressanten im Gedicht rein fiktional sind oder auf den empirischen Autor bezogen werden können. In der dritten Sektion werden die Fragen der »Modalität« diskutiert, also ob die Äußerungsformen der Adressanten tatsächlich einem `Sprechen`, allenfalls einem `Schreiben` oder gar `Singen` entsprechen. Die vierte und letzte Sektion »Personalität« zeigt die Bandbreite der möglichen Perspektiven auf den lyrischen Adressanten auf, mitunter aus medienkomparatistischer, genderwissenschaftlicher, übersetzungstheoretischer Perspektive.

Den Einstieg in die erste Sektion bildet ein Glossar von Rüdiger Zymner, das einen systematischen Ordnungsversuch lyrikologischer Beschreibungsbegriffe darstellt. Das Glossar expliziert deshalb nicht nur den Begriff des >Adressanten`, sondern stellt ihn in einen größeren Zusammenhang der Beschreibung von lyrischen Sprachgebilden vor dem Hintergrund der in Zymner (2009) vorgelegten Lyrikdefinition.

Neben diesem systematischen Ansatz soll die terminologische Diskussion um eine differenzierte und adäquate Terminologie in der Lyrikforschung aber auch aus historischer Perspektive betrachtet werden. Während zum >lyrischen Ich bereits viele Darstellungen vorliegen, sodass hier auf eine weitere Darstellung verzichtet wurde, ist das Konzept des ılyrischen Helden` außerhalb der Slawistik wenig bekannt. Der wissenschaftsgeschichtliche Beitrag von Christian Zehnder zeigt, wie Jurij Tynjanov den Begriff in den 1920er Jahren in Auseinandersetzung mit der Lyrik Aleksandr Bloks entwickelte. Nach Tynjanov ist der slyrische Held als eine maskenhaft im Text dargebotene Selbstporträtierung des Autors im Sinne eines mythischen Selbstbildes, einer literarischen Persönlichkeit zu ver- 
stehen. Zehnder erläutert den literatursoziologischen Impetus Tynjanovs, die imaginations- und zeichentheoretischen Implikationen und Probleme, die mit dessen Begriffsprägung einhergehen, wie auch die vom Konzept des slyrischen Helden aufgeworfenen ethischen Fragen im Detail und vollzieht aus wissenschaftsgeschichtlicher Perspektive auch die Um- und Fehldeutungen nach, die Tynjanovs Begriff im Laufe des Zwanzigsten Jahrhunderts erfahren hat.

Evelyn Dueck stellt die Frage nach dem Adressanten in Celans Spätwerk, das bisher erstaunlich wenig untersucht wurde. Als theoretisches Fundament wie auch als Abgrenzungsfolie dient ihr dazu die transgenerische Narratologie, die sich auch der Rolle der Adressanten in Lyrik zugewandt hat. Mit Blick auf Celans Gedichtband kommt Dueck zu dem Schluss, dass die `Sprechinstanzen « in Fadensonnen überraschend kohärent gestaltet sind. Anhand von Celans Lyrik verdeutlicht Dueck exemplarisch die Potentiale und Grenzen der transgenerischen Narratologie und deren lyrikanalytischen Inventars.

Ralph Müller geht in seinem Beitrag von der Beobachtung aus, dass in der gegenwärtigen Lyriktheorie weiterhin adressantenorientierte Konzeptionen von Lyrik vorherrschen. Exemplarisch verdeutlicht er dies an den lyrikologischen Arbeiten von Jonathan Culler, Klaus W. Hempfer und Jochen Petzold. Müller prüft diese Ansätze kritisch im Hinblick auf ihre interpretationstheoretischen und literaturdidaktischen Konsequenzen und kommt zu dem Ergebnis, dass es auf dem gegenwärtigen Stand der Debatte aus interpretationstheoretischer Sicht verfrüht sei, die Definition der Lyrik mit einer spezifischen Äußerungsstruktur und mit spezifischen Kommunikationsmodellen zu verknüpfen, und aus literaturdidaktischer Sicht sogar insgesamt ungünstig, typische Adressantenkonstellationen in Lyrik zu verallgemeinern.

Die zweite Sektion widmet sich den grundlegenden Fragen der >Fiktionalität $<$ bzw. >Nicht-Fiktionalitätı lyrischer Adressanten. Fabian Lamparts Beitrag stellt den lyrikologischen Begriff der >Autorfaktualität` (das heißt den Fall, dass der Autor selbst sspricht` und Tatsächliches oder Wirkliches mitteilt) ins Zentrum seiner Überlegungen. Der Beitrag bietet in dieser Hinsicht einen Überblick über die wichtigsten Positionen in der kontrovers geführten Debatte zur Frage, ob lyrische Texte Äußerungen von Autorinnen und Autoren sein können und ob darin NichtFiktives mitgeteilt werden kann. Anhand von drei Beispielfällen (Fleming, Goethe, Enzensberger) diskutiert Lampart mögliche Konsequenzen für die Interpretationspraxis und plädiert dafür, von einer dichotomen Unterscheidung von Fiktionalität und Faktualität abzusehen und eine differenzierende Sichtweise anzunehmen, die Mischverhältnisse verschiedener `Sprechweisen` rekonstruiert. So würden die Beispielfälle Adressanten aufweisen, die einerseits autorfaktuale Anteile zeigen, die zudem falsifizierbar sind, andererseits aufgrund ästhetischer 
Gestaltungsstrategien sich von realweltlichen und biographischen Kontexten distanzieren.

Dass historische Auffassungen über den Status der Adressanten produktiv für die Interpretation genutzt werden können, zeigt Peer Trilckes Beitrag, der dafür plädiert, die »historischen Publikationskontexte und deren Logiken zu berücksichtigen«, also direkt die Frage zu stellen, welche Zuschreibungen reale Leserinnen und Leser im historischen Rezeptionskontext vorgenommen haben dürften. Wie Trilcke an einem Fallbeispiel von Theodor Fontane darlegt, können Genre, Publikationskontext und Epoche gute Gründe liefern, dass der Sprecher eines Gedichts zu Tagesaktualitäten im Sinne eines >publizistischen Subjekts` mit Fontane identifiziert wurde.

Als mögliche lyrische Adressanten oder Adressaten sind im Gedicht auch Figuren zu bedenken. Wie Claudia Hillebrandt darlegt, hat die transdisziplinäre Figurenforschung der Figur in der Lyrik bislang wenig Aufmerksamkeit geschenkt. Hillebrandt konzentriert sich auf den engeren Bereich der Lyrik, der nicht durch die Begrifflichkeiten der Narratologie erfasst wird; das heißt, Grenzfälle zwischen Erzählen und der Lyrik (wie zum Beispiel die Ballade) werden nicht berücksichtigt. Dadurch wird der Blick auf lyrikologische Besonderheiten gegenüber fiktionalen Erzählungen gerichtet. So ist die Differenzierung von Figur und Person in der Lyrik kultur-, epochen- und genreabhängig relevant. Ebenso haben Figuren an der Lyrik quantitativ weniger Anteil und sind ihre Figurenkonzepte weniger ausgestaltet: So werden Figuren zum Teil nur durch die Verwendung von Pronomina oder durch schematisierte Figurenkonzepte konstituiert und sind deshalb semantisch schwach festgelegt. Aufgrund des gegenüber Erzähltexten stärker ausgeprägten Artefaktcharakters von Lyrik könne davon ausgegangen werden, dass lyrische Figuren weniger als menschliche Aktanten verstanden werden, sondern häufiger als Symptome von Kommunikationsabsichten oder als Symbole.

Dieter Burdorf stellt in seinem Beitrag anhand von Beispielen aus der modernen Lyrik (seit etwa 1800) fest, dass nicht-fiktionale Namen nicht nur in den Paratexten von Gedichten auftauchen (etwa in Widmungsadressen oder Vorworten), sondern in unterschiedlichen Funktionen auch in den Gedichten selbst anzutreffen sind. Er plädiert daher dafür, Lyrik nicht per se als fiktional aufzufassen, sondern anzuerkennen, dass durch die Nennung von realen Namen aus der Gegenwart des Autors reale Personen konstitutiven Anteil am Gedicht haben können.

Eine wichtige, aber bisher nicht geklärte Rolle für die Rekonstruktion von Adressanten spielen Datums- und Ortsangaben, die bei vielen Gedichten in den sogenannten >Peritexten ` auftauchen. Diese Angaben beziehen sich, wie Sonja Klimeks Beitrag zeigt, in unterschiedlicher Weise auf den Adressanten in der Textwelt und die Autorperson in ihrer Diskurswelt. Solcherart autobiographisch 
inszenierende Peritexte eignen sich dafür, die verbreitete Ansicht herauszufordern, hochartifizielle Texte könnten unter keinen Umständen eine Referenz auf textexterne Wirklichkeit etablieren. Klimek unterscheidet Fälle, in denen sich die Orts- oder Zeitangaben a) auf den Ort und den Zeitpunkt beziehen lassen, zu dem die sprachlichen Äußerungen des Gedichttextes (angeblich) getätigt werden, b) auf Ort und Datum der in der Textwelt dargestellten Situation oder c) auf die Verfertigung des lyrischen Artefaktes als Text. Bei diesen drei Fällen können Ortsund Zeitangaben in den Peritexten von Gedichten je unterschiedliche Funktionen im Zusammenspiel von Lyrik und Autobiographik erfüllen.

In einem engen Verhältnis zum fiktionalen oder nicht-fiktionalen Status des Adressanten steht in der dritten Sektion die Frage der Modalität, wie wir sie schon mit Bezug auf das Sprechen von Gedichten angesprochen haben. Was ändert sich, wenn Lyrik gesprochen, gedruckt oder gar gesungen wird? Vor diesem Hintergrund beantwortet Rüdiger Zymner in seinem Beitrag »Text und animistische Literaturwissenschaft « die im vorliegenden Band aufgeworfene Frage »Wer spricht im Gedicht? « klar und dezidiert mit »tatsächlich niemand! «. Diese Antwort ergibt sich aus einer konsequent medientheoretischen Perspektive: Zymner geht von der Beobachtung aus, dass in der Literaturwissenschaft zumeist Texte im Sinne von Schriftzeichengebilden untersucht werden, deren graphische Repräsentationsform durch mündlichkeitsmetaphorische Ausdrücke wie zum Beispiel `Sprechinstanz oder 〉Rede` in irreführender Weise verdeckt wird. Zymner plädiert dafür, solche Mündlichkeitsmetaphorik in der literaturwissenschaftlichen Metasprache zu vermeiden. Mündlichkeitsmetaphorik sei nur in Auseinandersetzung mit tatsächlich mündlich vorgetragener Lyrik adäquat, bei der Analyse von schriftlich fixierter Lyrik führe sie aber zu Verwirrung, weil dadurch in unnötiger Weise zusätzliche Entitäten postuliert würden.

Die Situation, dass man Lyrik im Vortrag hört, mag gegenüber der Rezeption durch Schrift seltener sein, aber die Aufnahmetechniken für Ton und Bild speichern diese flüchtigen Vortragssituationen für eine nachträgliche Analyse. Anhand von solchen Aufnahmen behandelt Frieder von Ammon in seinem Beitrag »Wer spricht beim Gedichtvortrag? « die Frage nach dem Zusammenhang von textinternem Adressanten und demjenigen, der das Gedicht tatsächlich artikuliert. Im Zentrum stehen Fälle, in denen textinterne Adressanten und tatsächlich vortragende Sprecher in unterschiedlicher Weise miteinander korrelieren. Auf dieser Grundlage lässt sich eine Typologie entwickeln, die darlegt, wie Dichter, die ihre eigenen Gedichte vortragen, die Differenz zwischen Adressant und Autor während des Vortrags entweder markieren oder überspielen.

Im Zusammenhang mit der Verleihung des Literaturnobelpreises an Bob Dylan im Jahr 2016 erinnerte man sich in manchen Feuilletons, dass Lyrik einst- 
mals zur Lyra gesungen wurde. Eric Achermann bereitet diesen nach wie vor aktuellen Bezug anhand der Stellung der Stimme beim Song auf. Im Gegensatz zur Genette'schen Stimme wird die Stimme bei Lyrics in der Regel durch einen (oder mehrere) Sänger tatsächlich realisiert. Achermann schlägt in dieser Hinsicht vor, `Songtexte` als geschriebene und lesbare Worte von gesungenen und gehörten `Lyrics zu unterscheiden. Damit sieht Achermann auch eine Grundlage, um >Lyrics` von dominant schriftlicher Lyrik abzugrenzen. Hier gelte es zwei Differenzierungen vorzunehmen, denn der Song lässt sich auf keinen Fall unabhängig von der Musik betrachten, und seine Songhaftigkeit zeichnet ihn gegenüber dem Lied aus.

Wie die abschließenden Studien zur Personalität der Lyrik in der vierten Sektion belegen, führt die Frage »Wer spricht im Gedicht? « immer wieder zu ergiebigen Auseinandersetzungen mit der Poetik des Gedichts, mit Formen der Selbstdarstellung, mit der Herausforderung, Adressanten in Übersetzungen zuzuordnen, aber auch mit Fragen der Gender-Forschung. Gaby Pailer zeigt in Margaretha Susanna von Kuntschs (1654-1717) Ode »Als gleiches Unglück mit dem fünftgebornen Söhnlein / dem kleinen Chrisander, oder C. K. den 22. November 1686. durch GOttes Verhängniß sich begab« die Form der Trauerdarstellung auf. Die Auseinandersetzung mit dem Adressanten im frühneuzeitlichen Gedicht erfordert im Falle Kuntschs insbesondere eine gendertheoretische Perspektive. Pailer geht hierzu auf Kuntschs Bezugnahme auf den Iphigenien-Mythos und den Topos der Undarstellbarkeit höchster Trauer bei Plutarch ein. Im Anschluss rekonstruiert sie die autopoietische Dimension der Ode im Zusammenspiel von Adressant und Autorinnenrolle und weist zum Abschluss ihres Beitrags darauf hin, dass das Verhältnis von Werk und AutorInnenposition in der Frühen Neuzeit aus der Perspektive des Gender-Konzeptes differenzierter als bisher betrachtet werden müsse.

Zwei Fallstudien beziehen sich auf das für die lyrische Moderne zentrale Werk Rilkes. Laura Catania untersucht die schwer fassbare Position und Haltung des Adressanten in Rilkes erster Duineser Elegie und zeigt einen kunsthistorischen und intermedialen Zusammenhang zur kubistischen Malerei auf. Jeanne Wagner widmet sich Rilkes späten, so genannten `Doppelgedichten`. Am Beispiel von »Das Füllhorn « und »Corne d’Abondance« - zwei Gedichte mit gleichbedeutenden Titeln, die Rilke zeitgleich auf Deutsch und auf Französisch verfasste - lässt sich zeigen, wie die Wahl der Sprache und der damit verbundenen Lyriktradition sich jeweils auf die Gestaltung der gedichtinternen Sprechinstanz auswirkt.

Esther Hools Beitrag zu Elke Erbs Übersetzungen der russischen Gedichte von Marina Zwetajewa greift hingegen den `üblichen` Fall zwischensprachlicher Lyrikvermittlung auf, bei dem Autorin und Übersetzerin nicht identisch sind, der aber dadurch im Hinblick auf den Adressanten der Übersetzung keineswegs 
einfach angelegt ist. Im Zentrum steht bei Hool die Auseinandersetzung mit den je eigenen Mitteln, mit denen Erb Klang und Struktur von Zwetajewas Lyrik in eine eigene deutsche, von der russischen Vorlage aber nicht unabhängige Form bringt und in diesem Prozess ein eigenes Textsubjekt der Übersetzerin schafft.

Wie Stefanie Heine in ihrem Beitrag darlegt, meldet sich in Sylvia Plaths posthum erschienenen Gedichtband Ariel ein »Ich« bzw. ein »I« zu Wort, das entgegen verbreiteter autobiographischer Lektüren von Plaths Werk - in den Gedichten schon nicht mehr unter den Lebenden weilt. Die Gedichte werden dadurch aber nicht zu einfachen Fiktionen der Fantastik, vielmehr liegt eine bewusste Gestaltung des sprachlichen Ichs vor, das Spuren von Sylvia Plaths Ich trägt, als fiktives `Text-Ich ‘ aber nicht mehr aus dem Reich des Lebenden spricht. Dieses Ich ist sich seiner Literarizität äußerst bewusst, wie etwa aus dem Spiel von vertikalem »I « und horizontalem Bindestrich hervorgeht.

Fabian Schwitters Beitrag bietet eine Fallstudie zur formexperimentellen Gegenwartslyrik Ulf Stolterfohts und zugleich eine negative Antwort auf die Ausgangsfrage dieses Sammelbands, insofern in Stolterfohts Bänden laut Schwitter niemand von nichts spreche. Schwitter zeigt, dass Stolterfohts mehrbändiger Zyklus formale und wörtliche Versatzstücke von Fachsprachen aufweist, angeordnet in geometrisch formatierten Textblöcken als Produktionsprinzip. Der Gegenstand der Rede, üblicherweise im Fokus von fachsprachlichen Diskursen, trete dabei hinter das Interesse für das Medium zurück. Insgesamt legen also die Präsentation des sprachlichen Materials und die Form nahe, dass die Gedichte nicht auf einen Adressanten, sondern auf Fachsprachen selbst zurückgehen.

Der vorliegende Band versteht sich als Impulsgeber für die begriffstheoretische Diskussion sowie die begriffsgeschichtliche Aufarbeitung und somit als Plädoyer und Diskussionsvorlage für die Etablierung einer systematischen Lyrikforschung, der Lyrikologie. Die Herausgeber danken dem Schweizerischen Nationalfonds für die großzügige finanzielle Förderung eines »International Exploratory Workshops « sowie für die Übernahme der Satz- und Open-Access-Publikationskosten.

\section{Literaturverzeichnis}

Ammon, Frieder von. »Lyrikologie«. Handbuch literarischer Rhetorik. Hg. Rüdiger Zymner. Berlin: de Gruyter, 2015. 221-242.

Anderegg, Johannes. »Das Gedicht« [1985]. Lyriktheorie. Texte vom Barock bis zur Gegenwart. Hg. Ludwig Völker. Durchgesehene und bibliographisch ergänzte Aufl. Stuttgart: Reclam, 2000. 429-436.

Ball, Hugo. Gedichte. Hg. Eckhard Faul. Göttingen: Wallstein, 2007. 
Benn, Gottfried. Gesammelte Werke in der Fassung der Erstdrucke. Vier Bde. Essays und Reden in der Fassung der Erstdrucke. Hg. Bruno Hillebrand. Frankfurt a. M.: Fischer, 1989.

Borkowski, Jan und Simone Winko. »Wer spricht das Gedicht? noch einmal zum Begriff lyrisches Ich und zu seinen Ersetzungsvorschlägen «. Lyrische Narrationen - narrative Lyrik.

Gattungsinterferenzen in der mittelalterlichen Literatur. Hg. Hartmut Bleumer und Caroline Emmelius. Berlin: de Gruyter, 2011. 43-77.

Burdorf, Dieter. Einführung in die Gedichtanalyse. 3. Aufl. Stuttgart: Metzler, 2015.

Culler, Jonathan. Theory of the Lyric. Cambridge, MA.: Harvard Univ. Press, 2015.

Fricke, Harald und Peter Stocker. »Lyrisches Ich«. Reallexikon der deutschen Literaturwissenschaft. Hg. Harald Fricke, Klaus Grubmüller, Jan-Dirk Müller und Klaus Weimar. Bd. 2. Berlin: de Gruyter, 2000. 509-511.

Genette, Gérard. Introduction à l'architexte. Paris: Seuil, 1979.

Gernhardt, Robert. Gesammelte Gedichte 1954-2004. Frankfurt a. M.: Fischer, 2005.

Gertken, Jan und Tilmann Köppe. »Fiktionalität«. Grenzen der Literatur. Hg. Simone Winko, Fotis Jannidis und Gerhard Lauer. Berlin: de Gruyter, 2009. 228-266.

Hamburger, Käte. Die Logik der Dichtung [1957]. 3. Aufl. Stuttgart: Klett, 1977. 217-232.

Hempfer, Klaus W. Lyrik. Skizze einer systematischen Theorie. Stuttgart: Franz Steiner, 2014. Hühn, Peter und Jens Kiefer. The Narratological Analysis of Lyric Poetry. Studies in English Poetry from the 16th to the 20th Century. Berlin: de Gruyter, 2005.

Jackson, Virginia und Yopie Prins. »General Introduction«. The Lyric Theory Reader. A Critical Introduction. Hg. Virginia Jackson und Yopie Prins. Baltimore, MD.: Johns Hopkins Univ. Press, 2014. 1-8.

Kayser, Wolfgang. »Wer erzählt den Roman?« Die Neue Rundschau 68 (1957): 444-459.

Killy, Walter. Elemente der Lyrik. München: Beck, 1972.

Kindt, Tom und Hans-Harald Müller. The Implied Author. Concept and Controversy. Berlin: de Gruyter, 2006.

Kindt, Tom und Hans-Harald Müller. "Six Ways Not to Save the Implied Author«. Style 45 (2011): 67-79.

Köppe, Tilmann und Jan Stühring. »Against Pan-Narrator Theories«. Journal of Literary Semantics 40 (2011): 59-80.

Kress, Gunther und Theo van Leeuwen. Multimodal Discourse. The Modes and Media of Contemporary Communication. London: Bloomsbury, 2017.

Lamping, Dieter. Das lyrische Gedicht. Definitionen zu Theorie und Geschichte der Gattung [1989]. 3. Aufl. Göttingen: Vandenhoeck \& Ruprecht, 2000.

Martínez, Matías. „Das lyrische Ich. Verteidigung eines umstrittenen Begriffs«. Autorschaft. Positionen und Revisionen. Hg. Heinrich Detering. Stuttgart: Metzler, 2002. 376-389.

Müller, Wolfgang G. »Das lyrisches Ich«. Handbuch Lyrik. Theorie, Analyse, Geschichte. Hg. Dieter Lamping. Stuttgart: Metzler, 2011. 56-58.

Müller-Zettelmann, Eva. Lyrik und Metalyrik. Theorie einer Gattung und ihrer Selbstbespiegelung anhand von Beispielen aus der englisch- und deutschsprachigen Dichtung. Heidelberg: Winter, 2000.

Müller-Zettelmann, Eva und Margarete Rubik (Hg.). Theory into Poetry. New Approaches to the Lyric. Amsterdam: Rodopi, 2005.

Müller-Zettelmann, Eva. »Lyrik und Narratologie«. Erzähltheorie transgenerisch, intermedial, interdisziplinär. Hg. Vera Nünning und Ansgar Nünning. Trier: Wissenschaftlicher Verlag Trier, 2002. 129-153. 
Nägele, Rainer. „Der Diskurs des andern. Hölderlins Ode ıStimme des Volks und die Dialektik der Aufklärung". Le pauvre Holterling. Blätter zur Frankfurter Ausgabe 4/5. Basel: Stroemfeld, 1980. 61-76.

Novak, Julia. Live Poetry. An Integrated Approach to Poetry in Performance. Amsterdam: Rodopi, 2011.

Petzold, Jochen. Sprechsituationen lyrischer Dichtung. Ein Beitrag zur Gattungstypologie. Würzburg: Königshausen \& Neumann, 2012.

Rajewsky, Irina O. Intermedialität. Tübingen: Francke, 2002.

Schönert, Jörg, Peter Hühn und Malte Stein. Lyrik und Narratologie. Text-Analysen zu deutschsprachigen Gedichten vom 16. bis zum 20. Jahrhundert. Berlin: de Gruyter, 2007.

Spinner, Kaspar H. Zur Struktur des lyrischen Ich. Frankfurt a. M.: Akad. Verlagsgesellschaft, 1975.

Susman, Margarete. Das Wesen der modernen deutschen Lyrik. Stuttgart: Strecker \& Schröder, 1910.

Vischer, Friedrich Theodor. Aesthetik oder Wissenschaft des Schönen. Dritter Theil: Die Kunstlehre. Zweiter Abschnitt. Die Künste. Fünftes Heft: Die Dichtkunst. Reutlingen: Mäcken, 1857.

Wolf, Werner. »The Lyric: Problems of Definition and a Proposal for Reconceptualisation«. Theory into Poetry. Hg. Margarete Rubik und Eva Muller-Zettelmann. Amsterdam: Rodopi, 2005. 21-56.

Zipfel, Frank. »Lyrik und Fiktion«. Handbuch Lyrik. Theorie, Analyse, Geschichte. Hg. Dieter Lamping. Stuttgart: Metzler, 2011. 162-166.

Zymner, Rüdiger. Lyrik. Umriss und Begriff. Paderborn: Mentis, 2009. 
